\title{
Selection Properties and Set-Valued Young Integrals of Set-Valued Functions
}

\author{
Mariusz Michta and Jerzy Motyl@
}

\begin{abstract}
The paper deals with some selection properties of set-valued functions and different types of set-valued integrals of a Young type. Such integrals are considered for classes of Hölder continuous or with bounded Young p-variation set-valued functions. Two different cases are considered, namely set-valued functions with convex values and without convexity assumptions. The integrals contain as a particular case setvalued stochastic integrals with respect to a fractional Brownian motion, and therefore, their properties are crucial for the investigation of solutions to stochastic differential inclusions driven by a fractional Brownian motion.
\end{abstract}

Mathematics Subject Classification. 26A33, 26A16, 26A45, 28B20, 47H04.

Keywords. Hölder-continuity, set-valued function, set-valued Young and Riesz p-variation, set-valued Young integral, selection, generalized Steiner center.

\section{Introduction}

Since the pioneering work of Aumann [6], the notion of set-valued integrals for multivalued functions has attracted the interest of many authors both from theoretical and practical points of view. In particular, the theory has been developed extensively, among others, with applications to optimal control theory, mathematical economics, theory of differential inclusions and set-valued differential equations, see e.g. $[1,4,13,22,26,37]$. Later, the notion of the integral for set-valued functions has been extended to a stochastic case where set-valued Itô and Stratonovich integrals have been studied and applied to stochastic differential inclusions and set-valued stochastic differential equations, see e.g., 
[23-25,33-35]. Moreover, concepts of set-valued integrals, both deterministic and stochastic, were used to define the notion of fuzzy integrals applied in the theory of fuzzy differential equations, e.g., [16,21,27,32]. On the other hand, in a single-valued case, one can consider stochastic integration with respect to non-semimartingale integrators such as the Mandelbrot fractional Brownian motion which has Hölder continuous sample paths ([31]). Such integrals can be understood in the sense of Young [38]. This kind of integrals have been developed and widely used in the theory of differential equations by many authors, see e.g., [8,12,18,19,28,30].

Furthermore, control and optimal control problems inspired the intensive expansion of differential and stochastic set-valued inclusions theory. Thus it seems reasonable to investigate also differential inclusions driven by a fractional Brownian motion and Young type integrals also. Recently, in [7] the authors considered a Young type differential inclusion, where solutions were understood as Young integrals of appropriately regular selections of multivalued right-hand side. A similar idea was used for a stochastic inclusion with a fractional Brownian motion in [29]. Both the Aumann and set-valued Itô integrals play a crucial role in the studies of properties of solution sets, e.g., $[2,3,17,22,23,37]$. Therefore, it is quite natural to introduce set-valued Young type integrals. Motivated by this, the aim of this work is to introduce some types of set-valued Young integrals and to investigate their properties, especially these which seem to be useful in the Young set-valued inclusions theory. Such set-valued integrals are considered for classes of Hölder continuous setvalued functions, set-valued functions having a bounded Young p-variation as well as for set-valued functions with a bounded Riesz p-variation. In the paper, we consider two different cases, namely set-valued functions with convex values and without convexity assumptions. The properties of the two integrals and selection theorems presented in the paper are significantly different. Such integrals contain as a particular case set-valued stochastic integrals with respect to a fractional Brownian motion. Therefore, in our opinion, their properties are crucial not only for the existence of solutions to stochastic differential inclusions and set-valued stochastic differential equations driven by a fractional Brownian motion but also for useful properties of their solution sets.

The paper is organized as follows. In Sect. 2, we define spaces of setvalued functions being Hölder continuous and spaces of set-valued functions of a finite Young or Riesz $p$-variation. Several basic relations between such spaces are given. Section 3 deals with the properties of sets of appropriately regular selections of such set-valued functions, both in convex and nonconvex cases. Finally, in Sect. 4, we introduce set-valued Young type integrals which are based on the sets of selections examined in Sect. 3. We shall give a number of properties of such set-valued integrals. Some of them are natural multivalued generalizations of those known from a single-valued Young integral theory. 


\section{Hölder-Continuous and Finite p-Variation Set-Valued Functions}

Let $(X,\|\cdot\|)$ be a Banach space. Let $T>0$ and $\beta \in(0,1]$. For every function $f:[0, T] \rightarrow X$ we define

$$
\|f\|_{\infty}=\sup _{t \in[0, T]}\|f(t)\|_{X} \text { and } M_{\beta}(f)=\sup _{0 \leq s<t \leq T} \frac{\|f(t)-f(s)\|_{X}}{(t-s)^{\beta}} .
$$

$\operatorname{By} \mathcal{C}^{\beta}(X)$ we denote the space of $\beta$-H ölder-continuous ( or shortly $\beta$-Hölder) functions with a finite norm

$$
\|f\|_{\beta}:=\|f\|_{\infty}+M_{\beta}(f) .
$$

It can be shown that $\left(\mathcal{C}^{\beta},\|\cdot\|_{\beta}\right)$ is a Banach space.

Let $C l(X)$ be a family of all nonempty and closed subsets of $X$. By $c l_{X}(A)$ we denote the closure in the norm topology of the set $A \subset X$. Let $\operatorname{Comp}(X)$ and Conv $(X)$ denote the families of all nonempty and compact, and nonempty compact and convex subsets of $X$, respectively. The Hausdorff metric $H_{X}$ in $\operatorname{Comp}(X)$ is defined by

$$
H_{X}(B, C)=\max \left\{\bar{H}_{X}(B, C), \bar{H}_{X}(C, B)\right\},
$$

where $\bar{H}_{X}(B, C)=\sup _{b \in B} \operatorname{dist}_{X}(b, C)=\sup _{b \in B} \inf _{c \in C}\|c-b\|_{X}$. If $X$ is separable, then the space $\left(\operatorname{Comp}(X), H_{X}\right)$ is a Polish space and $\left(\operatorname{Conv}(X), H_{X}\right)$ is its closed subspace. For $B, C, D, E \in \operatorname{Comp}(X)$ we have,

$$
H_{X}(B+C, D+E) \leq H_{X}(B, D)+H_{X}(C, E)
$$

where $B+C:=\{b+c: b \in B, c \in C\}$ denotes the Minkowski sum of $B$ and $C$. Moreover, for $B, C, D \in \operatorname{Conv}(X)$ the equality

$$
H_{X}(B+D, C+D)=H_{X}(B, C),
$$

holds, see e.g., [20] for details.

We use the notation

$$
\|A\|_{X}:=H_{X}(A,\{0\})=\sup _{a \in A}\|a\|_{X} \text { for } A \in \operatorname{Conv}(X) .
$$

Similarly, for a set-valued function $F:[0, T] \rightarrow \operatorname{Comp}(X)$ let

$$
\|F\|_{\beta}:=\|F\|_{\infty}+M_{\beta}(F)
$$

where

$$
\|F\|_{\infty}=\sup _{t \in[0, T]}\|F(t)\|_{X} \text { and } M_{\beta}(F)=\sup _{0 \leq s<t \leq T} \frac{H_{X}(F(t), F(s))}{(t-s)^{\beta}} .
$$

A set-valued function $F$ is said to be $\beta$-Hölder if $\|F\|_{\beta}<\infty$. By $\mathcal{C}^{\beta}(\operatorname{Comp}(X))$ we denote the space of all such set-valued functions. The space of $\beta$-Hölder setvalued functions having also convex values will be denoted by $\mathcal{C}^{\beta}(\operatorname{Conv}(X))$. Introducing metrics 


$$
\begin{aligned}
& H_{\beta}^{(1)}(F, G) \\
& \quad=\sup _{t \in[0, T]} H_{X}(F(t), G(t))+\sup _{0 \leq s<t \leq T} \frac{H_{X}(F(t)+G(s), G(t)+F(s))}{(t-s)^{\beta}}
\end{aligned}
$$

in $\mathcal{C}^{\beta}(\operatorname{Conv}(X))$ and

$$
\begin{aligned}
& H_{\beta}^{(2)}(F, G) \\
& \quad=\sup _{t \in[0, T]} H_{X}(F(t), G(t))+\sup _{0 \leq s<t \leq T} \frac{\left|H_{X}(F(t), F(s))-H_{X}(G(t), G(s))\right|}{(t-s)^{\beta}}
\end{aligned}
$$

in $\mathcal{C}^{\beta}(C o m p(X))$, one can prove the following result.

Proposition 1. Spaces $\left(\mathcal{C}^{\beta}(\operatorname{Conv}(X)), H_{\beta}^{(1)}\right)$ and $\left(\mathcal{C}^{\beta}(\operatorname{Comp}(X)), H_{\beta}^{(2)}\right)$ are complete metric spaces. Moreover, $\left(\mathcal{C}^{\beta}(\operatorname{Conv}(X)), H_{\beta}^{(2)}\right)$ is a closed subspace of the space $\left(\mathcal{C}^{\beta}(\operatorname{Comp}(X)), H_{\beta}^{(2)}\right)$.

Let $(E, d)$ be a metric space. For every $0 \leq a<b \leq T$, by $\Pi_{n}=\left\{t_{i}\right\}_{i=0}^{n}$ we denote a partition $a=t_{0}<t_{2}<\ldots<t_{n}=b$ of the interval $[a, b]$. For every function $f:[0, T] \rightarrow E$ and $1 \leq p<\infty$ we define its Young p-variation on $[a, b]$ by the formula

$$
\operatorname{Var}_{p}(f,[a, b])=\sup _{\Pi} \sum_{i=0}^{n}\left(d\left(f\left(t_{i-1}\right), f\left(t_{i}\right)\right)^{p}\right.
$$

and a Riesz p-variation on $[a, b]$ by the formula

$$
V_{p}(f,[a, b])=\sup _{\Pi} \sum_{i=0}^{n} \frac{\left(d\left(f\left(t_{i-1}\right), f\left(t_{i}\right)\right)^{p}\right.}{\left(t_{i}-t_{i-1}\right)^{p-1}}
$$

We denote $\operatorname{Var}_{p}(f,[0, T])$ simply by $\operatorname{Var}_{p}(f)$ and $V_{p}(f,[0, T]):=V_{p}(f)$, respectively. If $\operatorname{Var}_{p}(f)<\infty$ (resp., $V_{p}(f)<\infty$ ) we call $f$ a bounded Young (resp., Riesz) p-variation function. The class of all functions of bounded pvariations will be denoted by $B \operatorname{Var}_{p}([0, T], E)$ or $B V_{p}([0, T], E)$, respectively. In the sequel we denote spaces $B \operatorname{Var}_{p}([0, T], E)$ and $B V_{p}([0, T], E)$ simply by $B \operatorname{Var}_{p}(E)$ and $B V_{p}(E)$, respectively. If $\left(X,\|\cdot\|_{X}\right)$ is a Banach space, then $B \operatorname{Var}_{p}(X)$ or $B V_{p}(X)$ are Banach spaces, if considered with norms $\|f\|_{\operatorname{Var}_{p}}=$ $\sup _{t \in[0, T]}\|f(t)\|_{X}+\left(\operatorname{Var}_{p}(f)\right)^{1 / p}$ and $\|f\|_{V_{p}}=\sup _{t \in[0, T]}\|f(t)\|_{X}+\left(V_{p}(f)\right)^{1 / p}$, respectively.

We collect some properties of functions of bounded p-variations in the following theorem.

Theorem 1. ([11]). Let $f:[0, T] \rightarrow E$. Then, for every $1 \leq p<\infty$, the following conditions hold: 
(a) if $A C(E)$ denotes the class of all absolutely continuous functions and $\operatorname{Lip}(E)$ the set of all Lipschitz functions from $[0, T]$ to $E$, then, for every $1<p<q<\infty$, the inclusions

$$
\begin{aligned}
& \operatorname{Lip}(E) \subset B V_{q}(E) \subset B V_{p}(E) \subset A C(E) \subset B V_{1}(E) \\
& =B \operatorname{Var}_{1}(E) \subset B \operatorname{Var}_{p}(E) \subset B \operatorname{Var}_{q}(E)
\end{aligned}
$$

hold.

(b) For every $[a, b] \subset[0, T]$ and $a \leq t \leq b$ we have

$2^{1-p} \operatorname{Var}_{p}(f,[a, b]) \leq \operatorname{Var}_{p}(f,[a, t])+\operatorname{Var}_{p}(f,[t, b]) \leq \operatorname{Var}_{p}(f,[a, b])$

and

$$
V_{p}(f,[a, t])+V_{p}(f,[t, b])=V_{p}(f,[a, b]) .
$$

(c) $f \in B \operatorname{Var}_{p}(E)$ if and only if there exist a bounded increasing function $\phi:[0, T] \rightarrow R$ and a Hölder continuous map $g: \phi([0, T]) \rightarrow E$ of exponent $1 / p$, with a Hölder constant not greater than 1 and such that $f=g \circ \phi$,

(d) $f \in B V_{p}(E)$ if and only if there exist a bounded increasing function $\phi$ : $[0, T] \rightarrow R^{1}$ and a map $g: \phi([0, T]) \rightarrow E$ with $V_{1}(g,[a, b])=b-a$ and such that $f=g \circ \phi$,

(e) if $f \in B V_{p}(E)$, then $V_{1}(f,[a, b]) \leq(b-a)^{1-1 / p}\left(V_{p}(f,[a, b])\right)^{1 / p}$ for every $[a, b] \subset[0, T]$, (Jensen inequality).

(f) if $\left(f_{n}\right)$ is a sequence such that $\lim _{n \rightarrow \infty} d\left(f_{n}(t), f(t)\right)=0$ for every $t \in$ $[a, b]$ then $\operatorname{Var}_{p}(f,[a, b]) \leq \liminf _{n \rightarrow \infty} \operatorname{Var}_{p}\left(f_{n},[a, b]\right)$ and $V_{p}(f,[a, b]) \leq$ $\liminf _{n \rightarrow \infty} V_{p}\left(f_{n},[a, b]\right)$.

(g) if $X$ is a Banach space, then for every $f, g \in B V_{p}(X)$ and $\lambda \in[0,1]$ the inequality $V_{p}(\lambda f+(1-\lambda) g) \leq \lambda V_{p}(f,[0, T])+(1-\lambda) V_{p}(g,[0, T])$ holds.

(h) if $X$ is a reflexive Banach space and $f \in B V_{p}(X)$, then $f$ admits a strong derivative $f^{\prime}$ and $V_{p}(f,[a, b])=\int_{a}^{b}\left\|f^{\prime}(t)\right\|^{p} d t$, (Riesz theorem).

Let $(X,\|\cdot\|)$ be a Banach space and let $F:[0, T] \rightarrow \operatorname{Comp}(X)$ be a given set-valued function. In this case we can set $(E, d)=\left(\operatorname{Comp}(X), H_{X}\right)$ and define quantities $\operatorname{Var}_{p}(F)$ and $V_{p}(F)$ being a Young $p$-variation and Riesz $p$-variation for $F$, respectively.

By $B \operatorname{Var}_{p}(\operatorname{Comp}(X))$ (resp. $B V_{p}(\operatorname{Comp}(X))$ we denote the space of all set-valued functions from $[0, T]$ into $\operatorname{Comp}(X)$ having finite Young $p$-variations (resp., finite Riesz $p$-variations).

We set

$$
\|F\|_{\operatorname{Var}_{p}}:=\|F\|_{\infty}+\left(\operatorname{Var}_{p}(F)\right)^{1 / p}
$$

and

$$
\|F\|_{V_{p}}:=\|F\|_{\infty}+\left(V_{p}(F)\right)^{1 / p}
$$


Similarly, as in a single valued case, every $F \in \mathcal{C}^{\beta}(\operatorname{Comp}(X))$ satisfies $F \in$ $B \operatorname{Var}_{p}(\operatorname{Comp}(X))$ with $p=1 / \beta$, and

$$
\operatorname{Var}_{1 / \beta}(F) \leq T\left(M_{\beta}(F)\right)^{1 / \beta} \text { and }\|F\|_{\operatorname{Var}_{1 / \beta}} \leq \max \left\{1, T^{\beta}\right\}\|F\|_{\beta} .
$$

Moreover, since for every $F \in B V_{p}(\operatorname{Comp}(X)), p>1$ and $0 \leq s<t \leq T$ we have

$$
\frac{H_{X}(F(t), F(s))}{(t-s)^{1-1 / p}} \leq\left(V_{p}(F)\right)^{1 / p}
$$

then for $p>1$ we get

$$
\operatorname{Var}_{p /(p-1)}(F) \leq T\left(M_{(p-1) / p}(F)\right)^{p /(p-1)} \leq T\left(\left(V_{p}(F)\right)^{1 /(p-1)}\right.
$$

and

$$
\|F\|_{\operatorname{Var}_{p /(p-1)}} \leq \max \left\{1, T^{(p-1) / p}\right\}\|F\|_{(p-1) / p} \leq \max \left\{1, T^{(p-1) / p}\right\}\|F\|_{V_{p}} .
$$

\section{Selections of Nonconvex Valued Set-Valued Functions}

Let $F:[0, T] \rightarrow \operatorname{Comp}(X)$ be a measurable set-valued function. A measurable function $f:[0, T] \rightarrow X$ is called a measurable selection of $F$ if $f(t) \in F(t)$ for all $t \in[0, T]$. For $1 \leq p<\infty$, define the set

$$
S_{L^{p}}(F)=\left\{f \in L^{p}([0, T], X): f(t) \in F(t) \text { a.e. } \mathrm{t} \in[0, \mathrm{~T}]\right\} .
$$

$S_{L^{p}}(F)$ is a closed subset of $L^{p}([0, T], X)$. It is nonempty if $F$ is p-integrably bounded i.e., if there exists $g \in L^{p}\left([0, T]\right.$ such that $\|F(t)\|_{X} \leq g(t)$ for a.e. $t \in[0, T]$. In such a case there exists a sequence $\left(f_{n}\right) \subset S_{L^{p}}(F)$ such that $F(t)=\overline{\left\{f_{n}(t)\right\}_{n=1}^{\infty}}$ for all $t \in[0, T]$ (see [9]). The sequence $\left(f_{n}\right)$ is called an $L^{p_{-}}$ Castaing representation for $F$. For other properties of measurable set-valued functions and their measurable selections (see e.g. [5]).

Definition 1. Let $F:[0, T] \rightarrow \operatorname{Comp}\left(R^{d}\right)$ be a set-valued function. For $1 \leq$ $p<\infty$, define

$$
S_{\operatorname{Var}_{p}}(F):=\left\{f \in B \operatorname{Var}_{p}\left(R^{d}\right): f(t) \in F(t), t \in[0, T]\right\}
$$

and

$$
S_{V_{p}}(F):=\left\{f \in B V_{p}\left(R^{d}\right): f(t) \in F(t), t \in[0, T]\right\},
$$

the sets of selections of $F$ with a bounded Young p-variation and bounded Riesz p-variation, respectively.

First problem is connected with the nonemptiness of sets $S_{V_{a r}}(F)$ and $S_{V_{p}}(F)$.

Note, that in the case $p=1$ we have $B V_{1}\left(\operatorname{Comp}\left(R^{d}\right)\right)=B \operatorname{Var}_{1}$ $\left(\operatorname{Comp}\left(R^{d}\right)\right)$. When $p>1$ it seems that the question whether any compact valued set-valued function of finite Young $p$-variation admits a selection with 
a finite Young $p$-variation is still open (see e.g. Remark 8.1 in [10]). Therefore, we consider the more regular class $\mathcal{C}^{\beta}\left(\operatorname{Comp}\left(R^{d}\right)\right)$.

Let $F \in \mathcal{C}^{\beta}\left(C o m p\left(R^{d}\right)\right)$. Such set-valued functions need not admit any Hölder or even continuous selection (see Example 1 in Sect. 6 of Chap. I in [4] and Proposition 8.2 in [10]). However, the following selection theorem holds true (Theorem 1.2 in [7]).

Theorem 2. Let $\beta \in(0,1]$ and let $F:[0, T] \rightarrow \operatorname{Comp}\left(R^{d}\right)$ be $\beta$-Hölder setvalued function. Then, for any $p>1 / \beta$ and $x \in F(0)$, there exists a selection $f \in S_{\text {Varp }_{p}}(F)$ with $f(0)=x$ and such that

$$
\left(\operatorname{Var}_{p}(f)\right)^{1 / p} \leq M_{\beta}(F) .
$$

For $F \in \mathcal{C}^{\beta}\left(\operatorname{Comp}\left(R^{d}\right)\right)$ we define the following subset of $S_{\operatorname{Var}_{p}}(F)$

$$
S_{\operatorname{Var}_{p}}^{\beta}(F):=\left\{f \in S_{\operatorname{Var}_{p}}(F):\|f\|_{\operatorname{Var}_{p}} \leq\|F\|_{\beta}\right\} .
$$

Hence, by the theorem above, we have the following result used in Sect. 5 .

Corollary 1. Let $F \in \mathcal{C}^{\beta}\left(\operatorname{Comp}\left(R^{d}\right)\right)$ and $p>1 / \beta$. Then the sets $S_{V_{a r}}(F)$ and $S_{\operatorname{Var}_{p}}^{\beta}(F)$ are nonempty and closed subsets of $B \operatorname{Var}_{p}\left(R^{d}\right)$. The latter one is also bounded.

In order to obtain more regular selections we put our attention on a class $B V_{p}\left(\operatorname{Comp}\left(R^{d}\right)\right)$. For such class of set-valued functions we obtain not only nonemptiness of the set $S_{V_{p}}(F)$ but we get some additional properties of this set, which seem to be useful in the investigation of differental-integral inclusions with set-valued Young integrals.

Proposition 2. ([11]) Let $F:[0, T] \rightarrow \operatorname{Comp}\left(R^{d}\right)$ be a set-valued function. If $F \in B V_{p}\left(\operatorname{Comp}\left(R^{d}\right)\right)$ for some $1 \leq p<\infty$ then there exist a function $\phi \in$ $B V_{p}\left(R^{d}\right)$ and a sequence of equi-Lipschitzean functions $\left(g_{n}\right)_{n=1}^{\infty}$ with Lipschitz constants $L_{n} \leq 1$ such that taking $f_{n}:=g_{n} \circ \phi$, we have $V_{p}\left(f_{n},[a, b]\right) \leq$ $V_{p}(F,[a, b])$ for every $0 \leq a<b \leq T$ and $F(t)=\overline{\left\{f_{n}(t)\right\}_{n=1}^{\infty}}$ for every $t \in[0, T]$. The set $\left\{f_{n}\right\}_{n=1}^{\infty}$ is a $V_{p}$-Castaing representation for $F$.

From the above proposition it is easy to deduce the following result.

Theorem 3. Let $F_{1}, F_{2}:[0, T] \rightarrow \operatorname{Comp}\left(R^{d}\right)$ be set-valued functions. If $F_{1}, F_{2} \in$ $B V_{p}\left(\operatorname{Comp}\left(R^{d}\right)\right)$ for some $1 \leq p<\infty$ and $S_{V_{p}}\left(F_{1}\right)=S_{V_{p}}\left(F_{2}\right)$, then $F_{1}(t)=$ $F_{2}(t)$ for all $t \in[0, T]$.

Proposition 3. Let $F:[0, T] \rightarrow \operatorname{Comp}\left(R^{d}\right)$ be a set-valued function. If $F \in$ $B V_{p}\left(\operatorname{Comp}\left(R^{d}\right)\right)$ for some $1 \leq p<\infty$, then the set $S_{V_{p}}(F)$ is closed in the norm $\|\cdot\|_{V_{p}}$.

Proof. The set $S_{V_{p}}(F)$ is nonempty by Proposition 2. Let $\left(f_{n}\right) \subset S_{V_{p}}(F)$ be a sequence convergent to some function $f$ with respect to norm $\|\cdot\|_{V_{p}}$. Then $f(t) \in F(t)$ for every $t \in[0, T]$. Since $V_{p}(f+g)^{1 / p} \leq V_{p}(f)^{1 / p}+V_{p}(g)^{1 / p}$, 
then the real sequence $\left(V_{p}\left(f_{n}\right)\right)_{n=1}^{\infty}$ is Cauchy and therefore, bounded in $R^{1}$. Then there exists a constant $M>0$ such that $V_{p}\left(f_{n}\right) \leq M$ for every $n \geq 1$. But $V_{p}\left(f_{n}\right) \rightarrow V_{p}(f)$ and $\left\|f_{n}(t)-f(t)\right\|_{R^{d}} \leq\left\|f_{n}(0)-f(0)\right\|_{R^{d}}+\left(V_{p}\left(f_{n}-\right.\right.$ $f))^{1 / p} \cdot t^{1-1 / p} \leq \max \left\{1, T^{1-1 / p}\right\}\left\|f_{n}-f\right\|_{V_{p}} \rightarrow 0$ by Theorem $1(\mathrm{e})$. Then, using Theorem $1(\mathrm{f})$, we get $V_{p}(f) \leq M$ and therefore, $f \in S_{V_{p}}(F)$.

It is an open question if the analogues of Theorem 3 and Proposition 3 hold true for the larger class $B \operatorname{Var}_{p}\left(\operatorname{Comp}\left(R^{d}\right)\right)$.

The approximation property of the set $S_{V_{p}}(F)$ is placed in the "Appendix" as Theorem 7 , because it is not used in proofs of main results.

\section{Selections of Convex Valued Set-Valued Functions}

From a previous section we know that every set-valued function $F:[0, T] \rightarrow$ $\operatorname{Comp}\left(R^{d}\right)$ which has a finite Riesz p-variation admits a selection $f \in B V_{p}\left(R^{d}\right)$, Proposition 2. However, if $F \in \mathcal{C}^{\beta}\left(\operatorname{Comp}\left(R^{d}\right)\right)$, then it need not admit any $\beta$-Hölder selection. It has only selections with a finite Young $p$-variation, see Theorem 2. It seems that the question whether any compact valued set-valued function of finite Young $p$-variation admits a selection with a finite Young $p$ variation is still open (see. e.g., Remark 8.1 in [10]). The property of convexity of values of a given set-valued function is helpful in the problem.

For $F \in \mathcal{C}^{\beta}\left(\operatorname{Conv}\left(R^{d}\right)\right)$ and $G \in B \operatorname{Var}_{p}\left(\operatorname{Conv}\left(R^{d}\right)\right)$ we consider the set $S_{\beta}(F)$ of all $\beta$-Hölder selections of $F$, i.e., the set

$$
\left.S_{\beta}(F)=\left\{f \in \mathcal{C}^{\beta}\left(R^{d}\right): f(t) \in F(t), t \in[0, T]\right)\right\} .
$$

As before, the set $S_{\operatorname{Var}_{p}}(G)$ denotes the set of all selections of $G$ having a finite Young $p$-variation.

It is clear that for every $p=1 / \beta$ it holds $S_{\beta}(F) \subset S_{\operatorname{Var}_{p}}(F)$. Thus in order to show nonemptiness of the sets $S_{\beta}(F)$ for $F \in \mathcal{C}^{\beta}\left(\operatorname{Conv}\left(R^{d}\right)\right)$ and $S_{\operatorname{Var}_{p}}(G)$ for $G \in B \operatorname{Var}_{p}\left(\operatorname{Conv}\left(R^{d}\right)\right)$ we cannot apply Theorem 2. However, since both set-valued functons $F$ and $G$ have convex values, it is possible to use a generalized Steiner center (see e.g., [14]). Namely, let $C \in \operatorname{Conv}\left(R^{d}\right)$ and let $\sigma(\cdot, C): R^{d} \rightarrow R^{1}, \sigma(p, C)=\sup _{y \in C}<p, y>$ be a support function of $C$. Let $\Sigma$ denote the unit sphere in $R^{d}$ and let $V$ denote a Lebesgue measure of a closed unit ball $B(0,1)$ in $R^{d}$, i.e., $V=\pi^{d / 2} / \Gamma\left(1+\frac{d}{2}\right)$ with $\Gamma$ being the Euler function. Let $p_{V}$ be a normalized Lebesgue measure on $B(0,1)$, i.e., $d p_{V}=d p / V$. Let

$\mathcal{M}=\{\mu: \mu$ is a probability measure on $B(0,1)$ having the $C^{1}-$ density $d \mu / d p_{V}$ with respect to measure $\left.p_{V}\right\}$.

Let $\xi_{\mu}:=d \mu / d p_{V}$ and let $\nabla \xi_{\mu}$ denote the gradient of $\xi_{\mu}$. By $\omega$ we denote a Lebesgue measure on $\Sigma$. Then, for every $\mu \in \mathcal{M}$, the function $S t_{\mu}$ : 
$\operatorname{Conv}\left(R^{d}\right) \rightarrow R^{d}$ called a generalized Steiner center, and given by the formula

$$
S t_{\mu}(C)=V^{-1}\left(\int_{\Sigma} p \sigma(p, C) \xi_{\mu}(p) d \omega(p)-\int_{B(0,1)} \sigma(p, C) \nabla \xi_{\mu}(p) d p\right)
$$

has the following properties: for $A, B, C \in \operatorname{Conv}\left(R^{d}\right)$ and $a, b \in R^{1}$

$$
\begin{gathered}
S t_{\mu}(C) \in C, S t_{\mu}(a A+b B)=a S t_{\mu}(A)+b S t_{\mu}(B), \\
\left\|S t_{\mu}(A)-S t_{\mu}(B)\right\|_{R^{d}} \leq L_{\mu} \cdot H_{R^{d}}(A, B),
\end{gathered}
$$

where $L_{\mu}=d \max _{p \in \Sigma} \xi_{\mu}(p)+\max _{p \in B(0,1)}\left\|\nabla \xi_{\mu}(p)\right\|_{R^{d}}$ (see e.g., [15] ).

Then, for every $\mu \in \mathcal{M}$ and $G \in B \operatorname{Var}_{p}\left(\operatorname{Conv}\left(R^{d}\right)\right)$, the function $g_{\mu}(t):=S t_{\mu}(G(t))$ belongs to $S_{V a r_{p}}(G)$ and by (1) we have

$$
\operatorname{Var}_{p}\left(g_{\mu}\right) \leq L_{\mu} \operatorname{Var}_{p}(G) \text { and }\left\|g_{\mu}\right\|_{\operatorname{Var}_{p}} \leq \max \left\{1, L_{\mu}\right\}\|G\|_{\operatorname{Var}_{p}} .
$$

Moreover, by Theorem 3.4 from [14] it follows that $G \in B \operatorname{Var}_{p}\left(\operatorname{Conv}\left(R^{d}\right)\right)$ possesses a $\operatorname{Var}_{p}$-Castaing representation. More precisely, there exists a sequence $\left(\mu_{n}\right) \subset \mathcal{M}$ such that $G(t)=\overline{\left\{g_{\mu_{n}}(t)\right\}_{n=1}^{\infty}}$ for every $t \in[0, T]$.

Proposition 4. Let $G \in B \operatorname{Var}_{p}\left(\operatorname{Conv}\left(R^{d}\right)\right)$. Then the set $S_{V a r_{p}}(G)$ is closed and convex in $B \operatorname{Var}_{p}\left(R^{d}\right)$. Moreover, for any $G_{1}, G_{2} \in B \operatorname{Var}_{p}\left(\operatorname{Conv}\left(R^{d}\right)\right)$, $S_{V_{\text {arp }}}\left(G_{1}\right)=S_{\text {Var }_{p}}\left(G_{2}\right)$ if and only if $G_{1}(t)=G_{2}(t)$ for every $t \in[0, T]$.

Proof. The closedness and convexity of $S_{V_{a r}}(G)$ in $B \operatorname{Var}_{p}\left(R^{d}\right)$ are evident. Let $\left\{g_{\mu_{n}}\right\}_{n=1}^{\infty}$ be a $V a r_{p}$-Castaing representation for $G_{1}$ for some $\left\{\mu_{n}\right\}_{n=1}^{\infty} \subset$ $\mathcal{M}$. Then, for every $n \geq 1, g_{\mu_{n}} \in S_{\operatorname{Var}_{p}}\left(G_{1}\right)=S_{\operatorname{Var}_{p}}\left(G_{2}\right)$ and therefore, $g_{\mu_{n}}(t) \in G_{2}(t)$ for every $t \in[0, T]$ and $n \geq 1$. Since $G_{2}$ takes on closed values in $R^{d}$, then we have $G_{1}(t)=\overline{\left\{g_{\mu_{n}}(t)\right\}_{n=1}^{\infty}} \subset G_{2}(t)$ for every $t \in[0, T]$. The opposite inclusion can be proved in a similar way.

Let $F \in \mathcal{C}^{\beta}\left(\operatorname{Conv}\left(R^{d}\right)\right)$. Using a similar argumentation we deduce that for every $\mu \in \mathcal{M}$, the function $f_{\mu}(t):=S t_{\mu}(F(t))$ belongs to $S_{\beta}(F)$ and again by (1) we get

$$
M_{\beta}\left(f_{\mu}\right) \leq L_{\mu} M_{\beta}(F) \text { and }\left\|f_{\mu}\right\|_{\beta} \leq \max \left\{1, L_{\mu}\right\}\|F\|_{\beta} .
$$

Moreover, there exists a sequence $\left(\mu_{n}\right) \subset \mathcal{M}$ such that $F(t)=\overline{\left\{f_{\mu_{n}}(t)\right\}_{n=1}^{\infty}}$ for every $t \in[0, T]$. Similarly as above we obtain the following result:

Proposition 5. Let $F \in \mathcal{C}^{\beta}\left(\operatorname{Conv}\left(R^{d}\right)\right)$. Then the set $S_{\beta}(F)$ is closed and convex in $\mathcal{C}^{\beta}\left(R^{d}\right)$. Moreover, for $F_{1}, F_{2} \in \mathcal{C}^{\beta}\left(\operatorname{Conv}\left(R^{d}\right)\right), S_{\beta}\left(F_{1}\right)=S_{\beta}\left(F_{2}\right)$ if and only if $F_{1}(t)=F_{2}(t)$ for every $t \in[0, T]$.

In general, we cannot expect the boundedness of sets $S_{\beta}(F)$ in the space $\mathcal{C}^{\beta}\left(R^{d}\right)$ or sets $S_{\operatorname{Var}_{p}}(F)$ in $B \operatorname{Var}_{p}\left(R^{d}\right)$, even for a constant set-valued function $F$. Indeed, let $F(t) \equiv[0,1]$ for $t \in[0,1]$ and let us define for $n \geq 2$ 
functions $f_{n}$ by the formula

$$
f_{n}(t)=\left\{\begin{array}{cl}
0 & \text { for } 0 \leq t<1 / 2 \\
n t-n / 2 & \text { for } 1 / 2 \leq t<1 / 2+1 / n \\
1 & \text { for } 1 / 2+1 / n \leq t \leq 1
\end{array}\right.
$$

Then for every $t \in[0,1]$ and $n \geq 2, f_{n}(t) \in F(t), f_{n} \in \mathcal{C}^{\beta}\left(R^{1}\right)$ and for every $0<\beta \leq 1$ we have $\left\|f_{n}\right\|_{\beta} \geq 1+n^{\beta}$. Thus the set $S_{\beta}(F)$ is unbounded.

\section{Set-Valued Young Integrals}

At the beginning of this section we recall the notion of a Young integral in a single valued case introduced by Young [38]. For details see the excelent monography of Friz and Victoir [18]. Let $f:[0, T] \rightarrow R^{d}$ and $g:[0, T] \rightarrow R^{1}$ be given functions. For the partition $\Pi_{m}: 0=t_{0}<t_{1}<\ldots<t_{m}=T$ of the interval $[0, T]$ we consider the Riemann sum of $f$ with respect to $g$

$$
S\left(f, g, \Pi_{m}\right):=\sum_{i=1}^{m} f\left(t_{i-1}\right)\left(g\left(t_{i}\right)-g\left(t_{i-1}\right)\right) .
$$

Let $\left|\Pi_{m}\right|:=\max \left\{t_{i}-t_{i-1}: 1 \leq i \leq m-1\right\}$. Then the following version of Proposition 2.4 in [19] holds.

Theorem 4. Let $f \in B \operatorname{Var}_{p}\left(R^{d}\right)$ and $g \in \mathcal{C}^{\alpha}\left(R^{1}\right)$ where $1 / p+\alpha>1$. Then the limit

$$
\lim _{\left|\Pi_{m}\right| \rightarrow 0} S\left(f, g, \Pi_{m}\right)=: \int_{0}^{T} f d g
$$

exists and the inequality

$$
\left\|\int_{s}^{t} f d g-f(s)(g(t)-g(s))\right\|_{R^{d}} \leq C(\alpha, p)\left(\operatorname{Var}_{p}(f)\right)^{1 / p} M_{\alpha}(g)(t-s)^{\alpha}
$$

holds for every $0 \leq s<t \leq T$, where the constant $C(\alpha, p)$ depends only on $p$ and $\alpha$.

As an immediate consequence of (2) we have the following estimation.

Corollary 2. Let $f_{1}, f_{2} \in B \operatorname{Var}_{p}\left(R^{d}\right)$ and $g \in \mathcal{C}^{\alpha}\left(R^{1}\right)$ where $1 / p+\alpha>1$. Then

$$
\begin{aligned}
& \left\|\int_{0}^{.} f_{1} d g-\int_{0}^{.} f_{2} d g\right\|_{\alpha} \\
& \leq\left(\left\|f_{1}-f_{2}\right\|_{\infty}+C(\alpha, p)\left(\operatorname{Var}_{p}\left(f_{1}-f_{2}\right)\right)^{1 / p}\right) M_{\alpha}(g)\left(1+T^{\alpha}\right) .
\end{aligned}
$$


In the case $f \in \mathcal{C}^{\beta}\left(R^{d}\right)$ and $\alpha, \beta \in(0,1]$ with $\alpha+\beta>1$, one can express the Young integral by fractional derivatives. Namely, let $f_{0+}(t)=$ $(f(t)-f(0+)) I_{(0, T)}(t)$ and $f_{T-}(t)=(f(t)-f(T-)) I_{(0, T)}(t)$. The right-sided and left-sided fractional derivatives of order $0<\rho<1$ for the function $f:[0, T] \rightarrow R^{1}$ are defined by

$$
D_{0+}^{\rho} f(t)=\frac{1}{\Gamma(1-\rho)}\left(\frac{f(t)}{t^{\rho}}+\rho \int_{0}^{t} \frac{f(t)-f(s)}{(t-s)^{\rho+1}} d s\right)
$$

and

$$
D_{T-}^{\rho} f(t)=\frac{(-1)^{\rho}}{\Gamma(1-\rho)}\left(\frac{f(t)}{(T-t)^{\rho}}+\rho \int_{t}^{T} \frac{f(t)-f(s)}{(s-t)^{\rho+1}} d s\right) .
$$

Then one can prove the following result, see e.g., [36].

Proposition 6. Suppose that $g:[0, T] \rightarrow R^{1}, g \in \mathcal{C}^{\alpha}\left(R^{1}\right)$ and $f \in \mathcal{C}^{\beta}\left(R^{d}\right)$. Then the integral $\int_{0}^{T} f d g$ exists in the sense of Riemann and

$$
\int_{0}^{T} f d g=(-1)^{\rho} \int_{0}^{T} D_{0+}^{\rho} f_{0+}(t) D_{T-}^{1-\rho} g_{T-}(t) d t+f(0)(g(T)-g(0))
$$

for every $\rho \in(1-\alpha, \beta)$. Moreover, the following version of the inequality (2)

$$
\left\|\int_{t_{1}}^{t_{2}} f d g-f\left(t_{1}\right)\left(g\left(t_{2}\right)-g\left(t_{1}\right)\right)\right\|_{R^{d}} \leq C(\alpha, \beta) M_{\alpha}(g) M_{\beta}(f)\left(t_{2}-t_{1}\right)^{\alpha+\beta}
$$

holds for every $0 \leq t_{1}<t_{2} \leq T$, where $C(\alpha, \beta)$ depends only on $\alpha$ and $\beta$.

\subsection{Nonconvex Case}

Let $g \in C^{\alpha}\left(R^{1}\right)$ and let $F \in C^{\beta}\left(\operatorname{Comp}\left(R^{d}\right)\right)$ where $\alpha, \beta \in(0,1]$ and $\alpha+\beta>1$. By Corollary 1 , for any fixed $p>1 / \beta$ the sets $S_{\operatorname{Var}_{p}}(F)$ and $S_{\operatorname{Var}_{p}}^{\beta}(F)$ are nonempty and closed in $B \operatorname{Var}_{p}\left(R^{d}\right)$. If $1 / p+\alpha>1$, then we define a setvalued Young integral by the formula

$$
\int_{0}^{t} F d g:=c l_{R^{d}}\left\{\int_{0}^{t} f d g: f \in S_{\operatorname{Var}_{p}}(F)\right\} .
$$

Remark 1. Let $g \in C^{\alpha}\left(R^{l}\right)$ while $F \in C^{\beta}\left(\operatorname{Comp}\left(L\left(R^{l}, R^{d}\right)\right)\right)$. Repeating the proof of Theorem 1.2 in [7] it follows, that for every $p>1 / \beta$ and $g \in C^{\alpha}\left(R^{l}\right)$ there exists $f \in B \operatorname{Var}_{p}\left(L\left(R^{l}, R^{d}\right)\right)$ being a selection of $F$. Therefore, the definition of a set-valued Young integral given in formula (3) can be extended to the above case.

Proposition 7. For every $0 \leq s<t \leq T$, we have

$$
\sup _{0 \leq s<t \leq T} \frac{\left\|\int_{s}^{t} F d g\right\|_{R^{d}}}{(t-s)^{\alpha}}
$$




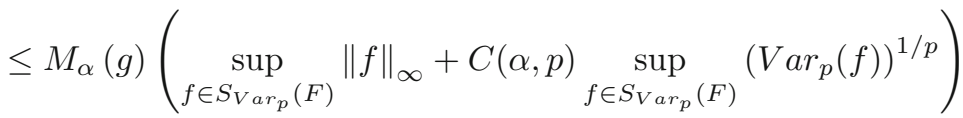

$$
\begin{aligned}
& \leq(1+C(\alpha, p)) M_{\alpha}(g) \sup _{f \in S_{\operatorname{Var}_{p}(F)}}\|f\|_{\operatorname{Var}_{p}} .
\end{aligned}
$$

Proof. Let $f \in S_{\operatorname{Var}_{p}}(F)$ and let $0 \leq s<t \leq T$. By (2) we have $\left\|\int_{s}^{t} f d g\right\|_{R^{d}} \leq\|f(s)\|_{R^{d}} M_{\alpha}(g)(t-s)^{\alpha}+C(\alpha, p)\left(\operatorname{Var}_{p}(f)\right)^{1 / p} M_{\alpha}(g)(t-s)^{\alpha}$.

Then, taking the supremum over $f \in S_{\operatorname{Var}_{p}}(F)$, we get

$$
\begin{aligned}
& \left\|\int_{s}^{t} F d g\right\|_{R^{d}} \\
& \leq M_{\alpha}(g)\left(\sup _{\left.f \in S_{\operatorname{Var}_{p}(F)}\|f(s)\|_{R^{d}}+C(\alpha, p) \sup _{f \in S_{\operatorname{Var}_{p}}(F)}\left(\operatorname{Var}_{p}(f)\right)^{1 / p}\right)(t-s)^{\alpha} .}\right.
\end{aligned}
$$

Hence we get estimations (4).

Let us note that in a single-valued case, i.e., $F=\{f\}$, an $\alpha$-Hölder continuity of a Young integral $[0, T] \ni t \rightarrow \int_{0}^{t} f d g \in R^{d}$ follows from Proposition 7 .

Let $B\left([0, T], R^{d}\right)$ denote a space of bounded functions from $[0, T]$ into $R^{d}$ with a sup-norm and let $H_{\infty}$ be a Hausdorff metric in a space $C l\left(B\left([0, T], R^{d}\right)\right)$. Similarly, by $H_{\operatorname{Var}_{p}}$ we mean a Hausdorff metric in the space $C l\left(B \operatorname{Var}_{p}\left(R^{d}\right)\right)$.

Theorem 5. Let $F_{1}, F_{2} \in \mathcal{C}^{\beta}\left(\operatorname{Comp}\left(R^{d}\right)\right)$. Let $p>1 / \beta$ and $1 / p+\alpha>1$. Then for every $0 \leq s<t \leq T$

$$
\begin{aligned}
& H_{R^{d}}\left(\int_{s}^{t} F_{1} d g, \int_{s}^{t} F_{2} d g\right) \leq M_{\alpha}(g)(t-s)^{\alpha} \\
& \times\left[H_{\infty}\left(S_{\operatorname{Var}_{p}}\left(F_{1}\right), S_{\operatorname{Var}_{p}}\left(F_{2}\right)\right)+C(\alpha, p) H_{\operatorname{Var}_{p}}\left(S_{\operatorname{Var}_{p}}\left(F_{2}\right), S_{\operatorname{Var}_{p}}\left(F_{2}\right)\right)\right](.5)
\end{aligned}
$$

Proof. Let $f_{1} \in S_{\operatorname{Var}_{p}}\left(F_{1}\right), f_{1} \in S_{\operatorname{Var}_{p}}\left(F_{2}\right)$. Then by (2) we have

$$
\begin{aligned}
& \left\|\int_{s}^{t} f_{1} d g-\int_{s}^{t} f_{2} d g\right\|_{R^{d}} \leq \\
& \leq\left(\left\|f_{1}(s)-f_{2}(s)\right\|_{R^{d}}+C(\alpha, p)\left(\operatorname{Var}_{p}\left(f_{1}-f_{2}\right)\right)^{1 / p}\right) M_{\alpha}(g)(t-s)^{\alpha} .
\end{aligned}
$$

Thus

$$
\begin{aligned}
& \operatorname{dist}_{R^{d}}\left(\int_{s}^{t} f_{1} d g, \int_{s}^{t} F_{2} d g\right)_{R^{d}} \leq \\
& \leq\left(\operatorname { d i s t } _ { \infty } \left(f_{1}, S_{\text {Var }_{p}}\left(F_{2}\right) \|_{\infty}+C(\alpha, p) \operatorname{dist}_{\text {Var }_{p}}\left(f_{1}, S_{\text {Var }_{p}}\left(F_{2}\right)\right) M_{\alpha}(g)(t-s)^{\alpha} .\right.\right.
\end{aligned}
$$

Hence we obtain

$$
\bar{H}_{R^{d}}\left(\int_{s}^{t} F_{1} d g, \int_{s}^{t} F_{2} d g\right)_{R^{d}}
$$




$$
\begin{aligned}
& \leq\left[H_{\infty}\left(S_{\operatorname{Var}_{p}}\left(F_{1}\right), S_{\operatorname{Var}_{p}}\left(F_{2}\right)\right)\right. \\
& \left.+C(\alpha, p) H_{\operatorname{Var}_{p}}\left(S_{\operatorname{Var}_{p}}\left(F_{1}\right), S_{\operatorname{Var}_{p}}\left(F_{2}\right)\right)\right] M_{\alpha}(g)(t-s)^{\alpha} .
\end{aligned}
$$

In a similar way we get the same estimation for $\bar{H}_{R^{d}}\left(\int_{s}^{t} F_{2} d g, \int_{s}^{t} F_{1} d g\right)_{R^{d}}$. This completes the proof.

In a single valued case, this theorem expresses continuity of the Young integration operator $B \operatorname{Var}_{p}\left(R^{d}\right) \ni f \rightarrow \int_{0}^{t} f d g \in R^{d}$.

Corollary 3. Let $F \in \mathcal{C}^{\beta}\left(\operatorname{Comp}\left(R^{d}\right)\right)$ and $p>1 / \beta$ with $1 / p+\alpha>1$. Then, for a set-valued function $[0, T] \ni t \rightarrow \int_{0}^{t} F d g \in C l\left(R^{d}\right)$, the inequality

$$
\begin{aligned}
& \left\|\int_{0}^{\cdot} F d g\right\|_{\alpha} \\
& \quad \leq M_{\alpha}(g)\left(1+T^{\alpha}\right)\left(\sup _{f \in S_{\operatorname{Var}_{p}(F)}}\|f\|_{\infty}+C(\alpha, p) \sup _{f \in S_{V a r_{p}}(F)}\|f\|_{V_{\text {ar }} p}\right) .
\end{aligned}
$$

holds.

Proof. For any $0 \leq s<t \leq T, \int_{0}^{t} F d g=\int_{0}^{s} F d g+\int_{s}^{t} F d g$ because the set $S_{\operatorname{Var}_{p}}(F)$ is interval-decomposable i.e.,

$$
\forall f_{1}, f_{2} \in S_{\operatorname{Var}_{p}}(F) \forall 0 \leq t \leq T \mathbb{1}_{[0, t]} \cdot f_{1}+\mathbb{1}_{[t, T]} \cdot f_{2} \in S_{\operatorname{Var}_{p}}(F) .
$$

Since

$$
\sup _{0 \leq s<t \leq T} \frac{H_{R^{d}}\left(\int_{0}^{t} F d g, \int_{0}^{s} F d g\right)}{(t-s)^{\alpha}} \leq \sup _{0 \leq s<t \leq T} \frac{\left\|\int_{s}^{t} F d g\right\|_{R^{d}}}{(t-s)^{\alpha}}
$$

then applying Proposition 7, we get

$$
\sup _{0 \leq s<t \leq T} \frac{H_{R^{d}}\left(\int_{0}^{t} F d g, \int_{0}^{s} F d g\right)}{(t-s)^{\alpha}} \leq(1+C(\alpha, p)) M_{\alpha}(g) \sup _{f \in S_{V^{a r_{p}}(F)}}\|f\|_{\text {Var }_{p}} .
$$

Therefore,

$$
\left\|\int_{0}^{\cdot} F d g\right\|_{\alpha} \leq \sup _{t \in[0, T]}\left\|\int_{0}^{t} F d g\right\|_{R^{d}}+\sup _{0 \leq s<t \leq T} \frac{\left\|\int_{s}^{t} F d g\right\|_{R^{d}}}{(t-s)^{\alpha}}
$$

and it is sufficient to apply Theorem 5 and Proposition 7 to get the desired estimation.

In general, the value of $H_{\operatorname{Var}_{p}}\left(S_{\operatorname{Var}_{p}}\left(F_{1}\right), S_{\operatorname{Var}_{p}}\left(F_{2}\right)\right)$ in the estimation (5) may be infinite. It is finite, if the definition of the set-valued Young integral given by formula $(3)$ is based on the set

$$
S_{\operatorname{Var}_{p}}^{\beta}(F)=\left\{f \in S_{\operatorname{Var}_{p}}(F):\|f\|_{\text {Var }_{p}} \leq\|F\|_{\beta}\right\} \text { for } p>1 / \beta
$$

instead of $S_{\text {Varp }_{p}}(F)$.

In such a case, we have the following result. 
Corollary 4. Let $F_{1}, F_{2} \in \mathcal{C}^{\beta}\left(\operatorname{Comp}\left(R^{d}\right)\right)$. Taking the set-valued integrals $\int_{s}^{t} F_{1} d g$ and $\int_{s}^{t} F_{2} d g$ with respect to sets $S_{\operatorname{Var}_{p}}^{\beta}, p>1 / \beta$ with $1 / p+\alpha>1$, the inequality

$H_{R^{d}}\left(\int_{s}^{t} F_{1} d g, \int_{s}^{t} F_{2} d g\right) \leq M_{\alpha}(g)(t-s)^{\alpha}(1+C(\alpha, p))\left(\left\|F_{1}\right\|_{\beta}+\left\|F_{2}\right\|_{\beta}\right)$.

holds for every $0 \leq s<t \leq T$.

\subsection{Convex Case}

Let $F:[0, T] \rightarrow \operatorname{Conv}\left(R^{d}\right)$. Let $\alpha, \beta \in(0,1]$ with $\alpha+\beta>1$. If $S_{\beta}(F) \neq \emptyset$ we define another type of a set-valued Young integral of $F$ over the interval $[0, t]$ , which is restricted to $\beta$-Hölder selections of $F$, i.e.,

$$
\int_{0}^{t} F d g:=c l_{R^{d}}\left\{\int_{0}^{t} f d g: f \in S_{\beta}(F)\right\} .
$$

In the whole Section 5.2 we consider integrals (6).

Remark 2. Formula (6) can be extended to the case $g \in C^{\alpha}\left(R^{l}\right)$ while $F \in$ $C^{\beta}\left(\operatorname{Conv}\left(L\left(R^{l}, R^{d}\right)\right)\right)$ by a slight modification. The space of linear bounded operators $L\left(R^{l}, R^{d}\right)$ coincides with a Banach space $M_{d \times l}$ of $d \times l$ matrices with the norm $\|a\|_{M_{d \times l}}^{2}=\sum_{i \leq d} \sum_{j \leq l} a_{i j}^{2}$ where $a=\left(a_{i j}\right)_{1 \leq i \leq d, 1 \leq j \leq l} \in M_{d \times l}$. Next the latter one is isometrically isomorphic with a finite dimensional Banach space $\left(R^{d l},\|\cdot\|_{R^{d l}}\right)$ where $\|\cdot\|_{R^{d l}}$ is an Euclidean norm. Let $\xi: M_{d \times l} \rightarrow R^{d l}$ denote this isometry and isomorphism . More precisely, for $a=\left(a_{i j}\right)_{1<i<d, 1<j<l} \in$ $M_{d \times l}$ let $a_{i} .=\left(a_{i 1}, a_{i 2}, \ldots, a_{i l}\right)$ denote the $i$-th row of $a$ for $i=1,2, \ldots, d$. Then, let $\xi(a)=\left(a_{1}, a_{2}, \ldots, a_{d}.\right) \in R^{d l}$. This is a suriective linear isometry. Next, let $S t_{\mu}: \operatorname{Conv}\left(R^{d l}\right) \rightarrow R^{d l}$ be a generalized Steiner centre with a Lipschitz constant $L_{\mu}$ for some fixed $\mu \in \mathcal{M}$ (see p.9). For every $A \in \operatorname{Conv}\left(L\left(R^{l}, R^{d}\right)\right)$ let us define a mapping $\tilde{S}_{\mu}(A):=\xi^{-1}\left(S t_{\mu}(\xi(A))\right.$. This mapping is single valued, i.e.

$$
\tilde{S}_{\mu}: \operatorname{Conv}\left(L\left(R^{l}, R^{d}\right)\right) \rightarrow L\left(R^{l}, R^{d}\right)
$$

and it is also a selection, i.e $\tilde{S}_{\mu}(A) \in A$ for every $A \in \operatorname{Conv}\left(L\left(R^{l}, R^{d}\right)\right)$. Moreover, we have

$$
\left\|\tilde{S}_{\mu}(A)-\tilde{S}_{\mu}(B)\right\|_{M_{d \times l}} \leq L_{\mu} H_{M_{d \times l}}(A, B)
$$

for every $A, B \in \operatorname{Conv}\left(L\left(R^{l}, R^{d}\right)\right)$. Hence $\tilde{S}_{\mu}$ is a Lipschitz selection. Finally, for $F \in C^{\beta}\left(\operatorname{Conv}\left(L\left(R^{l}, R^{d}\right)\right)\right)$ one can define a function $f_{\mu}:[0, T] \rightarrow L\left(R^{l}, R^{d}\right)$ as

$$
f_{\mu}(t):=\tilde{S}_{\mu}(F(t)) .
$$

By the properties above it follows that $f_{\mu} \in C^{\beta}\left(\left(L\left(R^{l}, R^{d}\right)\right)\right)$ and thus $S_{\beta}(F)$ $:=\left\{f \in C^{\beta}\left(\left(L\left(R^{l}, R^{d}\right)\right)\right): f(t) \in F(t), t \in[0, T]\right\}$ is nonempty. Therefore, the set-valued Young integral can be properly defined in this generalized case. 
Since $S_{\beta}(F) \subset S_{\operatorname{Var}_{p}}(F)$, where $p=1 / \beta$, then the integral defined by formula (6) is a subset of the integral (3). If $F \in \mathcal{C}^{\beta}\left(\operatorname{Conv}\left(R^{d}\right)\right)$, then the set-valued Young integral is a nonempty, closed and convex subset of $R^{d}$ for any $t \in[0, T]$ by Proposition 5. Similarly as Proposition 7 , one can prove the following.

Proposition 8. Let $F \in \mathcal{C}^{\beta}\left(\operatorname{Conv}\left(R^{d}\right)\right)$. Then, for every $0 \leq s<t \leq T$

$$
\begin{aligned}
& \sup _{0 \leq s<t \leq T} \frac{\left\|\int_{s}^{t} F d g\right\|_{R^{d}}}{(t-s)^{\alpha}} \\
& \leq M_{\alpha}(g)\left(\sup _{f \in S_{\beta}(F)}\|f\|_{\infty}+C(\alpha, \beta) \sup _{f \in S_{\beta}(F)} M_{\beta}(f) T^{\beta}\right) \\
& \leq M_{\alpha}(g) \sup _{f \in S_{\beta}(F)}\|f\|_{\beta}\left(1+C(\alpha, \beta) T^{\beta}\right) .
\end{aligned}
$$

Let $\mathcal{C}\left(R^{d}\right)$ denote the space of continuous functions on $[0, T]$ with values in $R^{d}$. We denote a Hausdorff distance in the space $C l\left(\mathcal{C}\left(R^{d}\right)\right)$ by $H_{\infty}$.

Theorem 6. Let $g \in \mathcal{C}^{\alpha}\left(R^{1}\right)$. Then, for every $\rho \in(1-\alpha, \beta)$, there exists a positive constant $C(\rho)$ such that for every $\theta \in(0,1], t \in[0, T]$ and every $F_{1}, F_{2} \in \mathcal{C}^{\beta}\left(\operatorname{Conv}\left(R^{d}\right)\right)$ the inequality

$$
\begin{aligned}
& H_{R^{d}}\left(\int_{0}^{t} F_{1} d g, \int_{0}^{t} F_{2} d g\right) \\
& \leq C(\rho)\left[H_{\infty}\left(S_{\beta}\left(F_{1}\right), S_{\beta}\left(F_{2}\right)\right)+\left(\sup _{f_{1} \in S_{\beta}\left(F_{1}\right)} M_{\beta}\left(f_{1}\right)\right.\right. \\
& \left.\left.+\sup _{f_{2} \in S_{\beta}\left(F_{2}\right)} M_{\beta}\left(f_{2}\right)\right) \theta^{\beta}\right] \theta^{-\rho} \\
& +M_{\alpha}(g) T^{\alpha} H_{\infty}\left(S_{\beta}\left(F_{1}\right), S_{\beta}\left(F_{2}\right)\right) .
\end{aligned}
$$

holds.

Remark 3. Let us note that the estimation in Theorem 6 does not follow by Theorem 5 . Indeed, the estimation on he right hand side in Theorem 6 concerns the Hausdorff distance between sets $S_{\beta}\left(F_{1}\right)$ and $S_{\beta}\left(F_{2}\right)$ for which $S_{\beta}\left(F_{1}\right) \subset S_{\operatorname{Var}_{p}}\left(F_{1}\right)$ and $S_{\beta}\left(F_{2}\right) \subset S_{\operatorname{Var}_{p}}\left(F_{2}\right)$ where $p=1 / \beta$. The distances $H_{\infty}\left(S_{\beta}\left(F_{1}\right), S_{\beta}\left(F_{2}\right)\right)$ and $H_{\infty}\left(S_{\operatorname{Var}_{p}}\left(F_{1}\right), S_{\operatorname{Var}_{p}}\left(F_{2}\right)\right)$ are not comparable in general. From this, it follows that the Hausdorff distance between set-valued integrals (6) is not comparable with the Hausdorff distance between integrals (3). Therefore, we cannot say which of results of Theorem 5 or Theorem 6 is more useful. Continuity of a set-valued Young integral can be applicable among other to investigation the existence and properties of solutions of differential inclusions. Applicability of continuity results presented above is strictly connected with the choice of a space to be more appropriate in the investigation of 
differential inclusions. Some results hold only for convex-valued multifunction, while other are true without convexity assumptions. For details see e.g. [4]

To prove Theorem 6 we need the following lemma.

Lemma 1. Let $g \in \mathcal{C}^{\alpha}\left(R^{1}\right)$. Then, for every $\rho \in(1-\alpha, \beta)$, there exists a positive constant $C(\rho)$ such that for every $f_{1}, f_{2} \in \mathcal{C}^{\beta}\left(R^{d}\right), t \in[0, T]$ and $\theta \in(0,1]$ the inequality

$$
\begin{aligned}
& \left\|\int_{0}^{t} f_{1} d g-\int_{0}^{t} f_{2} d g\right\|_{R^{d}} \leq C(\rho)\left[\left\|f_{1}-f_{2}\right\|_{\infty}+\left(M_{\beta}\left(f_{1}\right)+M_{\beta}\left(f_{2}\right)\right) \theta^{\beta}\right] \theta^{-\rho} \\
& \quad+\left\|f_{1}(0)-f_{2}(0)\right\|_{R^{d}}|g(T)-g(0)| .
\end{aligned}
$$

holds.

Proof. Let $\rho \in(1-\alpha, \beta)$ and $t \in[0, T]$. By Proposition 6 we get

$$
\begin{aligned}
& \left\|\int_{0}^{t} f_{1} d g-\int_{0}^{t} f_{2} d g\right\|_{R^{d}}=\left\|\int_{0}^{t}\left(f_{1}-f_{2}\right) d g\right\|_{R^{d}} \\
& \leq \int_{0}^{t}\left\|D_{0+}^{\rho}\left(f_{1}-f_{2}\right)_{0+}(s)\right\|_{R^{d}}\left|D_{t-}^{1-\rho} g_{t-}(s)\right| d s \\
& \quad+\left\|f_{1}(0)-f_{2}(0)\right\|_{R^{d}}|g(T)-g(0)| .
\end{aligned}
$$

Thus in order to obtain inequality (7), it is enough to prove the inequality

$$
\begin{aligned}
& \int_{0}^{t}\left\|D_{0+}^{\rho}\left(f_{1}-f_{2}\right)_{0+}(s)\right\|_{R^{d}}\left|D_{t-}^{1-\rho} g_{t-}(s)\right| d s \\
& \leq C(\rho)\left[\left\|f_{1}-f_{2}\right\|_{\infty}+\left(M_{\beta}\left(f_{1}\right)+M_{\beta}\left(f_{2}\right)\right) \theta^{\beta}\right] \theta^{-\rho} .
\end{aligned}
$$

Let $\theta \in(0,1)$. Then, for any $0 \leq s \leq t$, we have

$$
\begin{aligned}
& \left\|D_{0+}^{\rho}\left(f_{1}-f_{2}\right)_{0+}(s)\right\|_{R^{d}} \\
& \leq \frac{\rho}{\Gamma(1-\rho)}\left(\int_{0}^{s-\theta s} \frac{\left\|f_{1}(s)-f_{2}(s)-f_{1}(r)+f_{2}(r)\right\|_{R^{d}}}{(s-r)^{\rho+1}} d r\right. \\
& \left.+\int_{s-\theta s}^{s} \frac{\left\|f_{1}(s)-f_{2}(s)-f_{1}(r)+f_{2}(r)\right\|_{R^{d}}}{(s-r)^{\rho+1}} d r\right) \\
& \leq \frac{\rho}{\Gamma(1-\rho)}\left(\int_{0}^{s-\theta s} \frac{\left\|f_{1}(s)-f_{2}(s)\right\|_{R^{d}}}{(s-r)^{\rho+1}} d r+\int_{0}^{s-\theta s} \frac{\left\|f_{1}(r)-f_{2}(r)\right\|_{R^{d}}}{(s-r)^{\rho+1}} d r\right. \\
& +\int_{s-\theta s}^{s} \frac{\left\|f_{1}(s)-f_{1}(r)\right\|_{R^{d}}}{(s-r)^{\beta}} \frac{1}{(s-r)^{\rho+1-\beta}} d r \\
& \left.+\int_{s-\theta s}^{s} \frac{\left\|f_{2}(s)-f_{2}(r)\right\|_{R^{d}}}{(s-r)^{\beta}} \frac{1}{(s-r)^{\rho+1-\beta}} d r\right)
\end{aligned}
$$




$$
\begin{aligned}
& \leq \frac{\rho}{\Gamma(1-\rho)}\left(2\left\|f_{1}-f_{2}\right\|_{\infty} \int_{0}^{s-\theta s} \frac{1}{(s-r)^{\rho+1}} d r\right. \\
& \left.+\left(M_{\beta}\left(f_{1}\right)+M_{\beta}\left(f_{2}\right)\right) \int_{s-\theta s}^{s} \frac{1}{(s-r)^{\rho+1-\beta}} d r\right) .
\end{aligned}
$$

Since $\int_{0}^{s-\theta s}(s-r)^{-(\rho+1)} d r \leq \rho^{-1}(\theta s)^{-\rho}$ and the equality

$$
\begin{aligned}
& \int_{s-\theta s}^{s}(s-r)^{-(\rho+1-\beta)} d r=(\theta s)^{\beta-\rho}(\beta-\rho)^{-1} \text { holds, then we get } \\
& \left\|D_{0+}^{\rho}\left(f_{1}-f_{2}\right)_{0+}(s)\right\|_{R^{d}} \\
& \leq \frac{\rho}{\Gamma(1-\rho)}\left(2\left\|f_{1}-f_{2}\right\|_{\infty} \frac{1}{\rho(\theta s)^{\rho}}+\left(M_{\beta}\left(f_{1}\right)+M_{\beta}\left(f_{2}\right)\right) \frac{(\theta s)^{\beta-\rho}}{\beta-\rho}\right) .
\end{aligned}
$$

Moreover, the following estimation

$$
\left|D_{t-}^{1-\rho} g_{t-}(s)\right| \leq \frac{1-\rho}{\Gamma(\rho)} \int_{s}^{t} \frac{|g(s)-g(r)|}{(r-s)^{2-\rho}} d r \leq M_{\alpha}(g) \frac{1-\rho}{\Gamma(\rho)} \int_{s}^{t} \frac{d r}{(r-s)^{2-\rho-\alpha}}
$$

holds.

Since $\int_{s}^{t}(r-s)^{-(2-\rho-\alpha)} d r \leq t^{\rho+\alpha-1}(\rho+\alpha-1)^{-1}$ then we obtain

$$
\left|D_{t-}^{1-\rho} g_{t-}(s)\right| \leq \frac{(1-\rho) M_{\alpha}(g) t^{\rho+\alpha-1}}{\Gamma(\rho)(\rho+\alpha-1)} \text {. }
$$

Thus we get by (9) and (10),

$$
\begin{aligned}
& \int_{0}^{t}\left\|D_{0+}^{\rho}\left(f_{1}-f_{2}\right)_{0+}(s)\right\|_{R^{d}}\left|D_{t-}^{1-\rho} g_{t-}(s)\right| d s \\
& \leq \frac{\rho(1-\rho) M_{\alpha}(g) T^{\rho+\alpha-1}}{\Gamma(1-\rho) \Gamma(\rho)(\rho+\alpha-1)}\left(\frac{2\left\|f_{1}-f_{2}\right\|_{\infty}}{\rho \theta^{\rho}} \int_{0}^{t} \frac{d s}{s^{\rho}}\right. \\
& \left.+\left(M_{\beta}\left(f_{1}\right)+M_{\beta}\left(f_{2}\right)\right) \frac{\theta^{\beta-\rho}}{\beta-\rho} \int_{0}^{t} s^{\beta-\rho} d s\right)
\end{aligned}
$$

Let us take

$$
\begin{aligned}
& C_{1}(\rho)=\frac{2 \rho(1-\rho) M_{\alpha}(g) T^{\alpha}}{\Gamma(1-\rho) \Gamma(\rho)(\rho+\alpha-1) \rho(1-\rho)} \\
& C_{2}(\rho)=\frac{\rho(1-\rho) M_{\alpha}(g) T^{\alpha+\beta}}{\Gamma(1-\rho) \Gamma(\rho)(\rho+\alpha-1)(\beta-\rho)(\beta-\rho+1)}
\end{aligned}
$$

and

$$
C(\rho)=\max \left\{C_{1}(\rho), C_{2}(\rho)\right\} .
$$

We have by (11)

$$
\begin{aligned}
& \int_{0}^{t}\left\|D_{0+}^{\rho}\left(f_{1}-f_{2}\right)_{0+}(s)\right\|_{R^{d}}\left|D_{t-}^{1-\rho} g_{t-}(s)\right| d s \\
& \leq C(\rho)\left[\left\|f_{1}-f_{2}\right\|_{\infty}+\left(M_{\beta}\left(f_{1}\right)+M_{\beta}\left(f_{2}\right)\right) \theta^{\beta}\right] \theta^{-\rho}
\end{aligned}
$$


which is exactly (8).

Proof of Theorem 6. We obtain by Lemma 1, for any $f_{1} \in S_{\beta}\left(F_{1}\right), f_{2} \in$ $S_{\beta}\left(F_{2}\right), \rho \in(1-\alpha, \beta), t \in[0, T]$ and $\theta \in(0,1]$

$$
\begin{aligned}
& \left\|\int_{0}^{t} f_{1} d g-\int_{0}^{t} f_{2} d g\right\|_{R^{d}} \\
& \leq C(\rho)\left[\left\|f_{1}-f_{2}\right\|_{\infty}+\left(M_{\beta}\left(f_{1}\right)+M_{\beta}\left(f_{2}\right)\right) \theta^{\beta}\right] \theta^{-\rho}+\left\|f_{1}-f_{2}\right\|_{\infty} M_{\alpha}(g) T^{\alpha},
\end{aligned}
$$

where $C(\rho)$ is given by (12). Thus

$$
\begin{aligned}
& \operatorname{dist}_{R^{d}}\left(\int_{0}^{t} f_{1} d g, \int_{0}^{t} F_{2} d g\right) \\
& \leq C(\rho)\left[\operatorname{dist}_{\infty}\left(f_{1}, S_{\beta}\left(F_{2}\right)\right)+\left(\sup _{f_{1} \in S_{\beta}\left(F_{1}\right)} M_{\beta}\left(f_{1}\right)+\sup _{f_{2} \in S_{\beta}\left(F_{2}\right)} M_{\beta}\left(f_{2}\right)\right) \theta^{\beta}\right] \theta^{-\rho} \\
& +\operatorname{dist}_{\infty}\left(f_{1}, S_{\beta}\left(F_{2}\right)\right) M_{\alpha}(g) T^{\alpha} .
\end{aligned}
$$

Hence,

$$
\begin{aligned}
& \bar{H}_{R^{d}}\left(\int_{0}^{t} F_{1} d g, \int_{0}^{t} F_{2} d g\right) \\
& \leq C(\rho)\left[H_{\infty}\left(S_{\beta}\left(F_{1}\right), S_{\beta}\left(F_{2}\right)\right)+\left(\sup _{f_{1} \in S_{\beta}\left(F_{1}\right)} M_{\beta}\left(f_{1}\right)+\sup _{f_{2} \in S_{\beta}\left(F_{2}\right)} M_{\beta}\left(f_{2}\right)\right) \theta^{\beta}\right] \theta^{-\rho} \\
& +M_{\alpha}(g) T^{\alpha} H_{\infty}\left(S_{\beta}\left(F_{1}\right), S_{\beta}\left(F_{2}\right)\right) .
\end{aligned}
$$

The same estimation holds for $\bar{H}_{R^{d}}\left(\int_{0}^{t} F_{2} d g, \int_{0}^{t} F_{1} d g\right)$. This completes the proof.

Note, that the parameter $\theta \in(0,1]$ allows us to deduce the following corollary.

Corollary 5. Let $F_{n}, F \in C^{\beta}\left(\operatorname{Conv}\left(R^{d}\right)\right)$ be such that the sets $S_{\beta}\left(F_{n}\right), n \geq 1$ are uniformly bounded in $C^{\beta}\left(R^{d}\right)$ and $S_{\beta}(F)$ is bounded, then if

$$
H_{\infty}\left(S_{\beta}\left(F_{n}\right), S_{\beta}(F)\right) \rightarrow 0 \text {, as } n \rightarrow \infty,
$$

we have $\int_{0}^{t} F_{n} d g \rightarrow \int_{0}^{t} F d g$ in $\left(\operatorname{Conv}\left(R^{d}\right), H_{R^{d}}\right)$.

\section{Compliance with ethical standards}

Conflict of interest The authors declare that they have no conflict of interest.

Open Access. This article is licensed under a Creative Commons Attribution 4.0 International License, which permits use, sharing, adaptation, distribution and reproduction in any medium or format, as long as you give appropriate credit to the original author(s) and the source, provide a link to the Creative Commons licence, and indicate if changes were made. The images or other third party material in 
this article are included in the article's Creative Commons licence, unless indicated otherwise in a credit line to the material. If material is not included in the article's Creative Commons licence and your intended use is not permitted by statutory regulation or exceeds the permitted use, you will need to obtain permission directly from the copyright holder. To view a copy of this licence, visit http://creativecommons. org/licenses/by/4.0/.

\section{Appendix}

Theorem 7. Let $F:[0, T] \rightarrow \operatorname{Comp}\left(R^{d}\right)$ be a set-valued function such that $F \in B V_{p}\left(\operatorname{Comp}\left(R^{d}\right)\right)$ for some $1 \leq p<\infty$. Let $\left\{f_{m}\right\}_{m=1}^{\infty}$ be the $V_{p}$-Castaing representation of $F$ from Proposition 2. Then, for every $f \in S_{V_{p}}(F)$ and every $\epsilon>0$, there exist $n \geq 1$, a partition $\Pi_{n}: 0=t_{0}<t_{1}<\ldots<t_{n}=T$ and functions $f_{k_{0}}, \ldots, f_{k_{n}} \in\left\{f_{m}\right\}_{m=1}^{\infty}$ such that

$$
\left\|f-\sum_{j=0}^{n} \mathbb{1}_{\left[t_{j}, t_{j+1}\right)} \cdot f_{k_{j}}\right\|_{\infty}<\epsilon .
$$

Proof. Let $f \in S_{V_{p}}(F)$ be arbitrary taken. We deduce by Theorem 1(e) that $\|f(t)-f(s)\|_{R^{d}} \leq|t-s|^{1-1 / p} V_{p}(f)$. Therefore, for every $\epsilon>0$, there exists $\delta_{1}$ such that $\|f(t)-f(s)\|_{R^{d}} \leq \epsilon / 3$ for every $|t-s|<\delta_{1}$. Since $V_{p}\left(f_{m}\right) \leq V_{p}(F)$ by Proposition 2, then there exists $\delta_{2}$ such that $\left\|f_{m}(t)-f_{m}(s)\right\|_{R^{d}} \leq \epsilon / 3$ for every $|t-s|<\delta_{2}$ and $m=1,2, \ldots$. Let $\delta=\min \left\{\delta_{1}, \delta_{2}\right\}$ and let $n$ be such that $n \delta<T \leq(n+1) \delta$. Let us take a partition $\Pi_{n}: 0<\delta<2 \delta<\ldots<n \delta<T$. Since $f(t) \in F(t)=\overline{\left\{f_{m}(t)\right\}_{m=1}^{\infty}}$, then for every $k=0,1, \ldots, n$ there exists $m_{k}$ such that $\left\|f(k \delta)-f_{m_{k}}(k \delta)\right\|_{R^{d}}<\epsilon / 3$. Hence, for every $k=1,2, \ldots$ and each $t \in[k \delta, \min \{(k+1) \delta, T\}]$, we obtain

$$
\begin{aligned}
& \left\|f(t)-f_{m_{k}}(t)\right\|_{R^{d}} \leq\|f(t)-f(k \delta)\|_{R^{d}}+\left\|f(k \delta)-f_{m_{k}}(k \delta)\right\|_{R^{d}} \\
& +\left\|f_{m_{k}}(k \delta)-f_{m_{k}}(t)\right\|_{R^{d}} \leq \epsilon / 3+\epsilon / 3+\epsilon / 3=\epsilon .
\end{aligned}
$$

Thus,

$$
\begin{aligned}
& \left\|f-\sum_{k=0}^{n-1} \mathbb{1}_{[k \delta, \min \{(k+1) \delta, T\}]} \cdot f_{m_{k}}\right\|_{\infty} \\
& =\sup _{t \in[0, T]}\left\|f(t)-\sum_{k=0}^{n-1} \mathbb{I}_{[k \delta, \min \{(k+1) \delta, T\}]}(t) \cdot f_{m_{k}}(t)\right\|_{R^{d}} \\
& =\sup _{t \in \cup_{j=0}^{n}[j \delta, \min \{(j+1) \delta, T\}]}\left\|f(t)-\sum_{k=0}^{n-1} \mathbb{I}_{[k \delta, \min \{(k+1) \delta, T\}]}(t) \cdot f_{m_{k}}(t)\right\|_{R^{d}} \\
& =\max _{j=0, \ldots, n}\left\{\sup _{t \in[j \delta, \min \{(j+1) \delta, T\}]}\left\|f(t)-\sum_{k=0}^{n-1} \mathbb{I}_{[k \delta, \min \{(k+1) \delta, T\}]}(t) \cdot f_{m_{k}}(t)\right\|_{R^{d}}\right\} \\
& =\max _{j=0, \ldots, n}\left\{\sup _{t \in[j \delta, \min \{(j+1) \delta, T\}]}\left\|f(t)-f_{n_{j}}(t)\right\|_{R^{d}}\right\} \leq \epsilon .
\end{aligned}
$$


The following example shows that a similar approximation property with respect to $V_{p}$-variation norm need not hold true.

Example 1. Let $F:[0,1] \rightarrow \operatorname{Comp}\left(R^{1}\right)$ be defined by the formula $F(t) \equiv[0,1]$ for every $t \in[0,1]$. Then $\phi(t)=V_{1}(F,[0, t]) \equiv 0$. Let $\left\{f_{m}\right\}_{m=1}^{\infty}$ be an arbitrary Castaing representation of $F$ satisfying $V_{p}\left(f_{m}\right) \leq V_{p}(F)=0$ for every $m=$ $1,2, \ldots$. Then functions $f_{m}$ should be constant with $\overline{\left\{f_{m}(t)\right\}_{m=1}^{\infty}}=[0,1]$. Let $f \in S_{V_{p}}(F)$ be defined by the formula $f(t)=t$ for every $t \in[0,1]$. Let us take any $\epsilon<1 / 2$. For every partition $\Pi_{n}: 0=t_{0}<\ldots<t_{n}=1$, every $i=1,2 \ldots n$, and every functions $f_{k_{0}}, \ldots, f_{k_{n-1}} \in\left\{f_{m}\right\}_{m=1}^{\infty}$ we have

$$
\left\|f-\sum_{i=0}^{n-1} \mathbb{1}_{\left[t_{i}, t_{i+1}\right)} \cdot f_{k_{i}}\right\|_{V_{p}}=+\infty
$$

if at least two functions $f_{k_{i}}$ are different. If all constant functions $f_{k_{i}}$ are equal to some $f_{i}$, then

$$
\left\|f-\sum_{i=0}^{n-1} \mathbb{1}_{\left[t_{i}, t_{i+1}\right)} \cdot f_{i}\right\|_{V_{p}}=\left\|f-f_{i}\right\|_{V_{p}} \geq\left\|f-f_{i}\right\|_{\infty} \geq 1 / 2>\epsilon .
$$

\section{References}

[1] Ahmed, N.U.: Semigroup Theory with Applications to Systems and Control, Longman, Pitman Research Notes in Mathematics Series. Harlow, Essex (1991)

[2] Ahmed, N.U.: Optimal relaxed controls for nonlinear infinite dimensional stochastic differential inclusions. Marcel Dekker Lect. Notes Pure Appl. Math. 180, 1-19 (1994)

[3] Andres, J., Gabor, G., Górniewicz, L.: Acyclicity of solution sets to functional inclusions. Nonlinear Anal. 49, 671-688 (2002)

[4] Aubin, J.P., Cellina, A.: Differential inclusions. Springer, Berlin (1984)

[5] Aubin, J.P., Frankowska, H.: Set-Valued Analysis. Birkhäuser, Boston (1990)

[6] Aumann, R.J.: Integrals of set-valued functions. J. Math. Anal. Appl. 12, 1-12 (1965)

[7] Bailleul, I., Brault, A., Coutin, L.: Young and rough differential inclusions, HAL Id: hal-02148440, https://hal.archives-ouvertes.fr/hal-02148440, arXiv:1812.06727v2 [math.CA] (2019)

[8] Cass, T., Hairer, M., Litterer, C., Tindel, S.: Smoothness of the density for solutions to Gaussian rough differential equations. Ann. Probab. 43(1), 188-239 (2015)

[9] Castaing, C.: Sur l'existence des sections séparément mesurables et séparément continues d'une multi-application, Séminaire d'Analyse Convexe, Univ. des Sci. et Techniques du Languedoc Montpellier (1975) Expose No. 14

[10] Chistyakov, V.V., Galkin, O.E.: On maps of bounded $p$-variation with $p>1$. Positivity 2, 19-45 (1998) 
[11] Chistyakov, V.: Selections of bounded variation. J. Appl. Anal. 10(1), 1-82 (2004)

[12] Coutin, L., Qian, Z.: Stochastic analysis, rough paths analysis and fractional Brownian motions. Probab. Theory Relat. Fields 122, 108-140 (2002)

[13] Debreu, G.: Integration of correspondences. In: Proceedings of Fifth Berkeley Symposium on Mathematics Statist. and Probability, vol. II. Part I, pp. 351-372 (1966)

[14] Dentcheva, D.: Regular Castaing representations of set-valued functions with applications to stochastic programming. SIAM J. Optim. 10(3), 732-749 (2006)

[15] Dentcheva, D.: Differentiable selections and Castaing representations of multifunctions. J. Math. Anal. Appl. 371-396, 223 (1998)

[16] Diamond, P., Kloeden, P.: Metric Spaces of Fuzzy Sets: Theory and Applications. World Scientific, Singapore (1994)

[17] Djebali, S., Górniewicz, L., Ouahab, A.: Solutions sets for differential equations and inclusions. Series in Nonlinear Analysis Application. De Gruyter GmbH, Berlin, Boston (2013)

[18] Friz, P.K., Victoir, N.B.: Multidimensional Stochastic Processes as Rough Paths: Theory and Applications. Cambridge Studies in Advanced Mathematics (2010)

[19] Friz, P.K., Zhang, H.: Differential equations driven by rough paths with jumps. J. Differ. Equ. 264, 6226-6301 (2018)

[20] Hu, S., Papageorgiou, N.S.: Handbook of Multivalued Analysis. Vol. 1. Theory. Kluwer Academic Publishers, Dordrecht (1997)

[21] Kaleva, O.: The Cauchy problem for fuzzy differential equations. Fuzzy Sets Syst. 35, 389-396 (1990)

[22] Kisielewicz, M.: Differential Inclusions and Optimal Control. Kluwer Academic Publishers, Dordrecht (1991)

[23] Kisielewicz, M.: Stochastic Differential Inclusions and Applications. Springer, New York (2013)

[24] Kisielewicz, M., Michta, M.: Weak solutions of set-valued stochastic differential equations. J. Math. Anal. Appl. 473, 1026-1052 (2019)

[25] Kisielewicz, M., Motyl, J.: Selection theorems for set-valued stochastic integrals. Stoch. Anal. Appl. 37(2), 243-270 (2019)

[26] Lakshmikantham, V., Gnana Bhaskar, T., Vasundhara Devi, J.: Theory of Set Differential Equations in a Metric Space. Cambridge Scientific Publishers, Cambridge (2006)

[27] Lakshmikantham, V., Tolstonogov, A.A.: Existence and interrelation between set and fuzzy differential equations. Nonlinear Anal. 55, 255-268 (2003)

[28] Lejay, A.: Controlled differential equations as Young integrals: a simple approach. J. Differ. Equ. 249, 1777-1798 (2010)

[29] Levakov, A.A., Vas'kovskii, M.M.: Existence of solutions of stochastic differential inclusions with standard and fractional Brownian motions. Transl. Differ. Uravn. Differ. Equ. 51(8), 991-997 (2015)

[30] Lyons, T.: Differential equations driven by rough signals. Rev. Mat. Iberoam. 14, 215-310 (1998) 
[31] Mandelbrot, B., Van Ness, J.W.: Fractional Brownian motions, fractional noises and applications. SIAM Rev. 10, 422-437 (1968)

[32] Michta, M.: Set-valued stochastic integrals and fuzzy stochastic equations. Fuzzy Sets Syst. 177, 1-19 (2011)

[33] Michta, M.: On connections between stochastic differential inclusions and setvalued stochastic differential equations driven by semimartingales. J. Differ. Equ. 262, 2106-2134 (2017)

[34] Michta, M., Motyl, J.: Differentiable selections of set-valued functions and their applications. Nonlinear Anal. 66, 536-545 (2007)

[35] Michta, M., Motyl, J.: Martingale problem to Stratonovich stochastic inclusion. Nonlinear Anal. 71, e1307-e1311 (2009)

[36] Samko, S.G., Kilbas, A.A., Marichev, O.I.: Fractional Integrals and Derivatives, Theory and Applications. Gordon and Breach Science Publishers, Yverdon (1993)

[37] Tolstonogov, A.A.: Differential Inclusions in a Banach Space. Kluwer Academic Publishers, Dordrecht (2000)

[38] Young, L.S.: An inequality of the Hölder type connected with Stieltjes integration. Acta Math. 67, 251-282 (1936)

Mariusz Michta and Jerzy Motyl

Faculty of Mathematics, Computer Science and Econometrics

University of Zielona Góra

Szafrana $4 \mathrm{a}$

65-516 Zielona Gora

Poland

e-mail: m.michta@wmie.uz.zgora.pl;

j.motyl@wmie.uz.zgora.pl

Received: May 21, 2020.

Accepted: September 21, 2020.

Publisher's Note Springer Nature remains neutral with regard to jurisdictional claims in published maps and institutional affiliations. 